\title{
Design and realization of hardware-in-the-loop simulation system for pulsed laser fuze
}

\author{
Zhilin $\mathrm{GaO}^{1, \mathrm{a}}$,Huimin Chen ${ }^{1, \mathrm{~b}}$, Kerong Cai ${ }^{2, \mathrm{c}}$ \\ ${ }^{1}$ Science and Technology on Electromechanical Dynamic Control Laboratory, Beijing Institute of \\ Technology, Beijing 100081, China \\ ${ }^{2}$ Shanghai Electro-Mechanical Engineering Institute, Shanghai 201109, China \\ aopzhilin@163.com, b laserchm@126.com, ${ }^{\text {c}}$ caikerong@163.com
}

Keywords: Laser Fuze; Hardware-in-the-loop Simulation; Echo Signal Solve; Echo Signal Simulation; Echo Signal Calibration

\begin{abstract}
Laser fuze has advantages of anti electromagnetic interference and high accuracy of measuring distance, will become an important development direction of future fuze. This paper constructs the hardware-in-the-loop simulation system of pulsed laser fuze. Solved laser echo characteristic parameters of different encounter conditions based on the research of the environmental factors, target characteristics and the missile target encounter conditions etc. And take the characteristic parameters as the simulation parameters of laser echo. With the help of the echo signal simulator to simulate the processing of missile target encounter. The echo signal calibration system is used to calibrate the output laser power of the echo signal simulator. The experimental results show that: under the condition of laboratory, the system can realize the accurate simulation of the laser echo signal as well as has a certain reference value for the design of laser fuze.
\end{abstract}

\section{Introduction}

Pulsed laser fuze needs regular inspection and maintenance, wasting a lot of manpower and material resources with the traditional field target test, the cost is relatively high. Therefore, how to simulate the pulsed laser fuze actual working environment, and carries on the reliability of detection is very necessary. Pulsed laser fuze hardware-in-the-loop simulation system for fuze test under the condition of laboratory provides a new idea and solution, how to simulate pulse laser echo signal change fast and accurate in the process of missile target encounter is the key to the system performance. And the change of laser echo signal is mainly reflected in the signal pulse width and peak power of laser, signal pulse width can be simulated by the controller, and the simulation of laser peak power usually have different kinds of methods. Study and put forward the electric attenuation combined with the electric delay method to design echo signal simulator, and according to different conditions of the missile target encounter process to solve the laser fuze echo signal parameters, then the parameters is downloaded to the echo signal simulator to implementation of echo simulation.

\section{The principle and composition}

Pulsed laser fuze hardware-in-the-loop simulation system is composed of the echo signal solver, the echo signal simulator, the echo signal calibration system, the simulation control system, and the principle block diagram is shown as Fig.1. The echo signal solver is used to solve the echo signal characteristic parameters. The echo signal simulator receives the fuze products emission laser signal as the working time sequence signal of the hardware-in-the-loop simulation system, and according to the output echo characteristic parameters of the echo signal solver to simulate the laser echo signal of missile target encounter process, the simulate laser echo signal is received by the receiving system of fuze product. The echo signal calibration system is used to calibrate the output laser signal power of the echo signal simulator. The simulation control system is responsible to 
control the working of the hardware-in-the-loop simulation system.

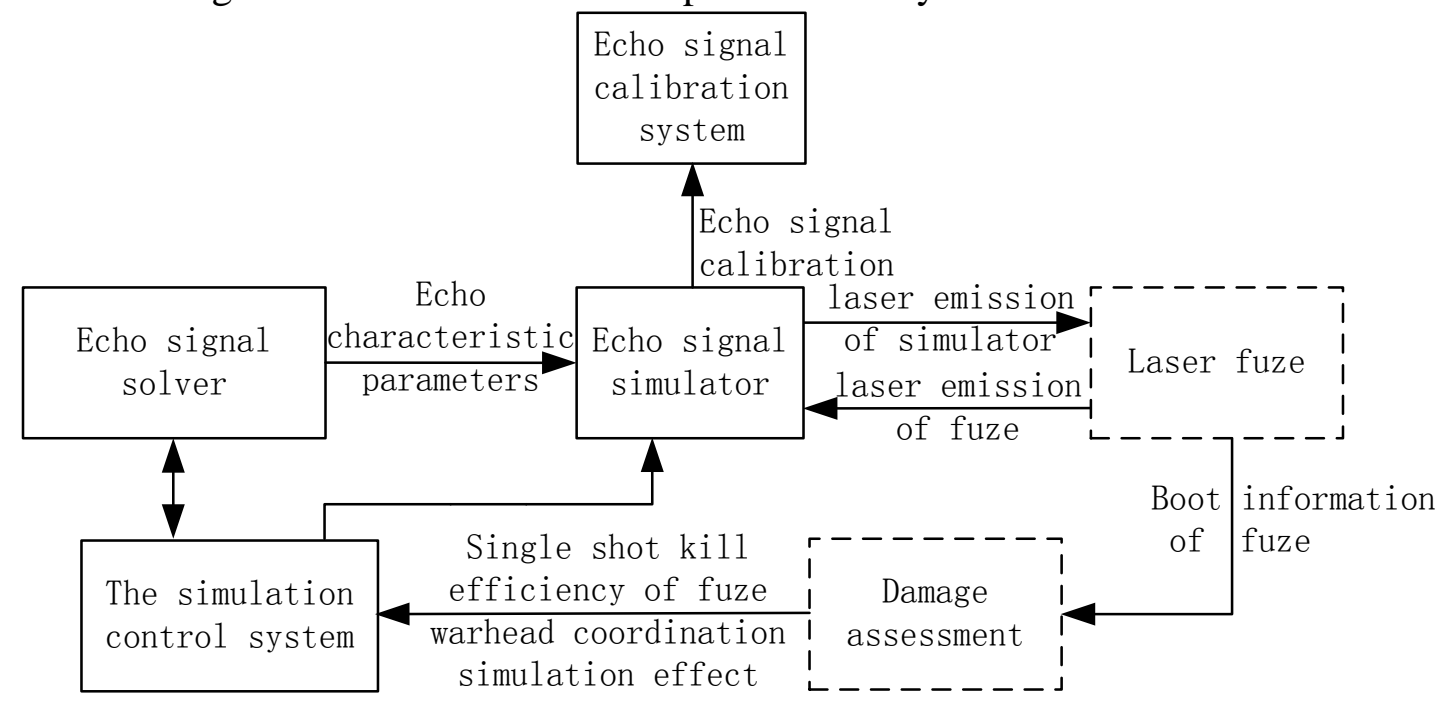

Fig.1. Principle block diagram of hardware-in-the-loop simulation system

\section{Design of the simulation control system}

The simulation control system is the total control component of the hardware-in-the-loop simulation system, as shown in Fig.1, the simulation control system controls the laser echo signal solver and makes it to output files which reflect the echo signal characteristic parameters. Then the simulation control system control the work of the echo signal simulator, and send the system self checking command to confirm that the system is working properly. When the system is working normally, the simulation control system will download the echo signal solver output files to the echo signal simulator. Finally, the simulation control system makes the fuze products working, and sends start the simulation commands to the echo signal simulator, when the echo signal simulator receives the start simulation command, it starts the simulation process under the action of the fuze sequence signal. The simulation control system will send the simulation end directive to the echo signal simulator while the fuze products send the detonation command to the simulation control system, and then ending the simulation process.

\section{Design of the laser echo signal solver}

Through analysis and research on the influence of different target, cloud, rain, snow, dust and other environmental factors to echo signal characteristics[1], and according to the target BRDF property[2] the laser echo signal solver can get the echo signal characteristic parameters. When pulsed laser fuze receiver field of view angle is greater than the emission beam divergence angle, its ranging formula[3] to the diffuse reflecting of big target can be expressed by Eq. (1)

$$
P_{r}=P_{t} \tau_{t} \tau_{r} \tau_{a}^{2} A_{r} \rho \cos \varphi / \pi R^{2}
$$

Where $P_{t}$ is the emission peak power of laser fuze, $P_{r}$ is the received peak power of laser fuze, $\tau_{t}$ is the transmitting optical system transmittance, $\tau_{r}$ is the receiving optical system transmittance, $A_{r}$ is the effective light receiving window area, $\rho$ is the target diffuse reflection coefficient, $\varphi$ is the angle between the optical axis of the target surface normal and the laser emission, $R$ is the target distance.

As the Eq.(1) shows, the laser echo power is related with the laser fuze system parameters and the atmospheric conditions, according to the Eq.(1) we can find the relationships between the laser echo signal power, the target distance and the atmospheric conditions.

The relationship between the target distance and the echo time delay can be expressed by Eq. (2), where $R$ is the target distance, $\Delta t$ is the echo time delay, $c$ is the speed of light in air. 
According to Eq. (2) we can get the relationship between the echo time delay and the target distance.

$$
R=\frac{\Delta t}{2} c
$$

According to the factors which are influence to the echo signal features above, by Visual C++ platform to achieve the building of the echo signal solver. With the help of OpenGL tool to achieve the visualization function, and then the echo signal solver software platform can be got. The echo signal solver software structure diagram is shown as Fig.2. The missile and target before the intersection is shown as Fig.3 and the missile and target encounter is shown as Fig.4.

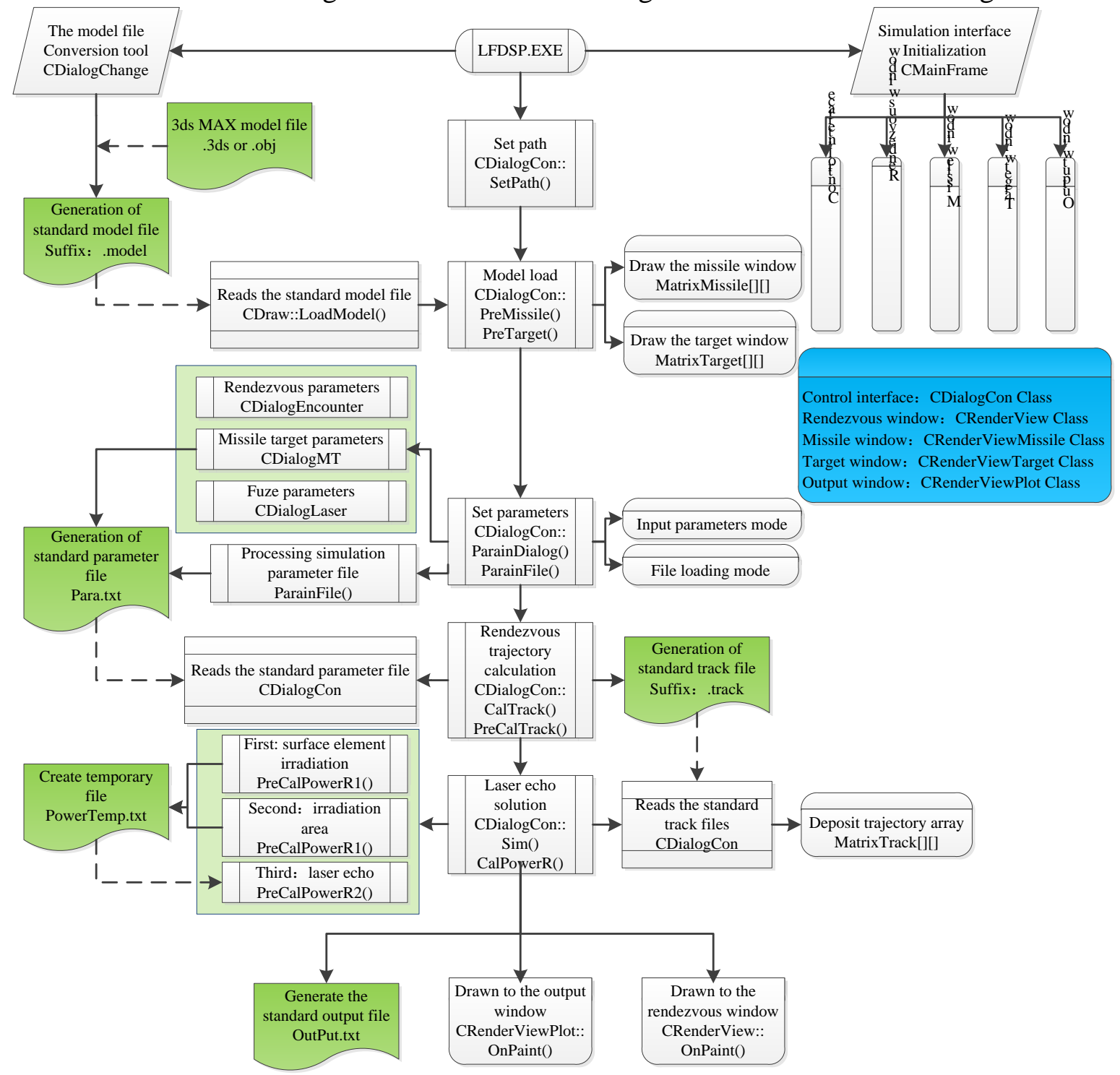

Fig.2. Diagram of echo signal solver software structure

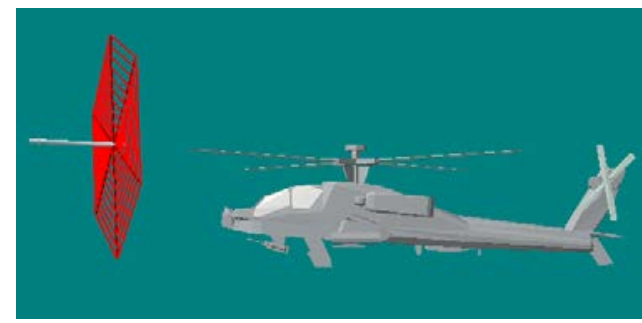

Fig.3. The missile and target before the intersection

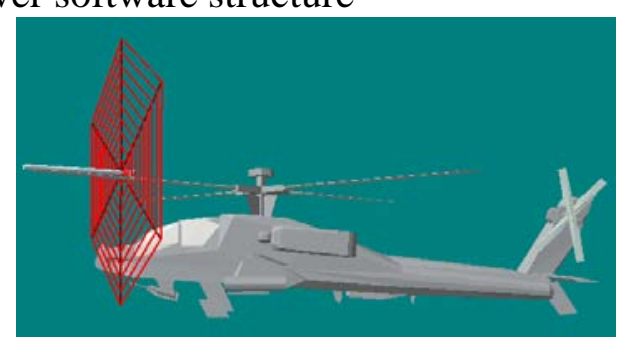

Fig.4. The missile and target encounter 


\section{Design of the laser echo signal simulator}

Under the control of the simulation control system, the laser echo signal simulator begin to operate by using the echo signal characteristic parameters and the fuze sequence signal which are output by the echo signal solver. The emission signal of laser fuze product is feed into the receiving window of the echo signal simulator through optical fiber and is converted into electric signal by pulse laser receiving circuit under the action of the beam shaping system. Then, the electric signal will be as the starting signal and the sequence of hardware-in-the-loop simulation system for synchronous signal. Under the action of the sequence signal, the echo signal simulator generate laser echo signal of simulation through the electric control attenuation device, the electric control delay device, the pulsed laser diode drive circuit, laser diode and beam shaping system. Then, the simulation echo signal will be feed into the fuze receiving window by optical fiber and realize the characteristics of laser fuze echo dynamic reiteration in the process of missile target encounter. The echo signal simulator principle diagram is shown as Fig.5.

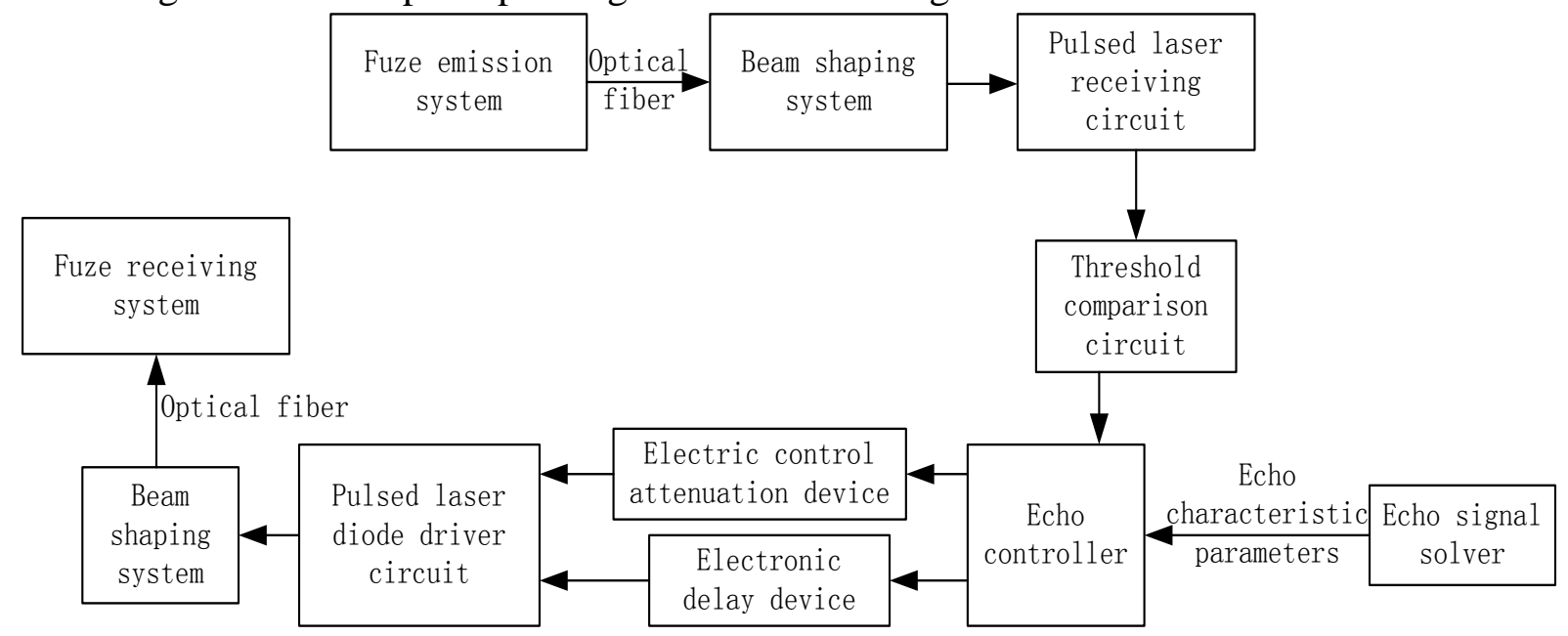

Fig.5. Composition principle graph of echo signal simulator

The echo signal simulator needs to simulate the laser echo power change and there are many methods to simulate laser echo power, such as crystal absorption method[4], photoelectric technology adjustment method, polarization attenuation method[5], absorption type variable attenuation method[6], mechanical adjustment aperture method etc. .

\section{Design of the laser echo signal calibration system}

The echo signal calibration system will calibrate the output laser power of the echo signal simulator. It is necessary to calibrate the output laser power while the supply power voltage to pulse laser diode driving circuit is different, as the laser echo signal power is simulated by the electric attenuation method. Making it satisfied to the relationship between laser echo power and missile target distance. When the pulse frequency $(10 \mathrm{kHz})$ and pulse width (50ns) of the driving signal to the pulse laser diode is fixed, using PM100D of THORLABS corporation to calibrate the output laser power of SPL PL85 laser of OSRAM corporation. By using the dynamometer to test the output peak power when the supply power voltage of the pulse laser diode driving circuit is different. The experimental result is shown as Fig.6 in the processing of Matlab. As shown in the calibration curve, pulse laser diode output power is positive linear relationship to its supply power voltage. 


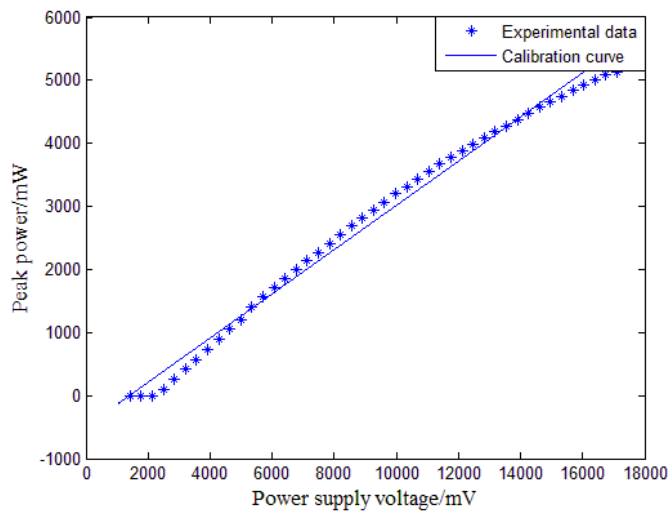

Fig.6. Relationship between supply voltage and the laser peak power

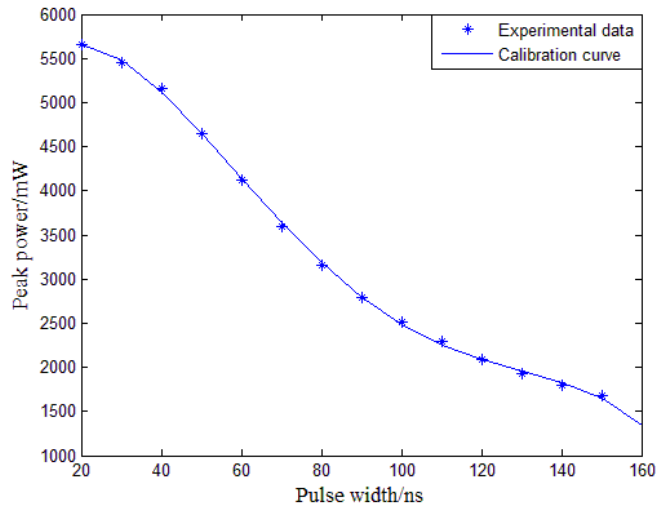

Fig.7. Relationship between pulse width and peak power of laser

The laser pulse width of the driving signal also has influence on the output power of the laser. To do experiment of several typical pulse width to test its affect to the output laser pulse peak power. When the pulse frequency $(10 \mathrm{kHz})$ and pulse laser diode driving circuit supply voltage (15V) is fixed, the relationship between pulse width and peak power of laser shown as Fig.7. The experimental result is shown as Fig.7 in the processing of Matlab. As shown in the calibration curve, laser output power is negative quadratic curve relationship to its pulse width.

\section{Conclusion}

Through the design and realization of hardware-in-the-loop simulation system for pulsed laser fuze, a set of perfect pulsed laser fuze testing device is established. And a complete set of control system software is developed. The system can conduct a comprehensive test of pulsed laser fuze and analysis the simulation result detailed. Using the electric attenuation combined with the electric delay method to design the echo signal simulator. It can simulate the echo signal power and the distance of simulation accurately. The system has a certain reference value.

\section{Acknowledgement}

In this paper, the research was supported by the National Defense Foundation of China (Grant No. 9140C360202130C36129).

\section{References}

[1] Biao Wang, Jiaxuan Lin, Guangde Tong. Simulation of laser beam scattering from complex targets[J]. GUIDANCE\&FUZE, 2012, 33(4): 24-30. In Chinese

[2] Leihong Zhang, Liujie Sun, Xiuhua Ma. Influence of bidirectional reflection distribution function of target satellite surface on echo in laser tracking[J]. Infrared and Laser Engineering, 2012, 41(8): 2048-2052. In Chinese

[3] Zhanzhong Cui, Shihe Song, Lixin Xu. The principle of proximity fuze[M]. Beijing: Beijing Institute of Technology Press, 2006. In Chinese

[4] Keyan Dong, Shoufeng Tong, Ying Ding, et al. Design of energy character simulation system for laser guidance hardware-in-the-loop simulation test[J]. Infrared and Laser Engineering, 2010, 39(S): 450-453. In Chinese

[5] Hongpeng Zhao, Yanfang Liu, Weiwei Liang, et al. Design of a high-precision continuous dynamic laser energy attenuation device and the measure to calibrate the precision of the device[J]. Infrared and Laser Engineering, 2013, 42(S1): 143-148. In Chinese

[6] Jiang Wang, Wei Lin, Peng Wang, et al. Analysis of laser energy chain in the hardware-in-the-loop simulation system of semi-active laser guidance[J]. Infrared and Laser Engineering, 2011, 40(7): 1230-1233. In Chinese 